\title{
Smoothed surface gradients for panel methods
}

\author{
J. D’Elía*, M. Storti, S. Idelsohn \\ Centro Internacional de Métodos Computacionales en Ingeniería (CIMEC) INTEC (UNL-CONICET), Güemes 3450, 3000-Santa Fe, Argentina
}

Accepted 18 November 1999

\begin{abstract}
A weak form to compute the dipolar and monopolar surface gradients, related to a low-order panel method, is shown. The flow problem is formulated by means of a three-dimensional potential model and the discretization is based on Morino's formulation for the perturbation velocity potential. On the body surface, this representation reduces to a boundary integral equation with the source (or monopolar) and the doublet (or dipolar) densities. The first of the two is found by application of the boundary flow condition, and the second one is the unknown over the body surface. A lower panel method is used for the analytic integrations of both the monopolar and dipolar influence coefficients. The surface velocity field is computed after solving the linear system, with a strong and a weak form of the Stokes theorem, which is oriented to fairly non-structured panel meshes. The proposed method is validated by comparing the numerical results with analytical ones for an isolated sphere and includes a prediction over a car-like configuration. (C) 2000 Elsevier Science Ltd. All rights reserved.
\end{abstract}

Keywords: Potential flows; Surface velocities; Boundary elements

\section{Introduction}

Panel methods are widely accepted in the aerospace and naval industry for calculating potential flows [1,2]. In some problems, a two-dimensional (2D) approach is sufficient, for instance, flows past multicomponent airfoils, infinite cascade, ground effects and wind tunnels $[3,4]$ while in other cases a three-dimensional (3D) approach is necessary. The 3D flows problem in these areas have special features that distinguish from other types [5]. In the first place, the body shapes are often quite complicated and, then, the methods applicable only to smooth shapes are neither fully useful nor are those whose computing costs increase rapidly with the boundary complexity. Also, the flow magnitudes are often very sensitive to small details of the body shape and the difference between good and bad aero/ hydro dynamic surfaces can be quite subtle; therefore a useful method must be able to discriminate between rather similar shapes. Often, it is desired to know not only the solution on the boundary but also its surface gradient. The most widely used approach to its computation in panel methods, is based on the use of the integral representation related with the formulation employed, but for the Morino's formulation [6] a rather simpler device was proposed by

\footnotetext{
* Corresponding author. Tel.: + 54-342-4559175; fax: + 54-3424550944.

E-mail address: rngtm@arcride.edu.ar (J. D’Elía).
}

Maître [7]. In particular, this author had shown that a numerical scheme for the doublet surface gradient based on a special form of the Stokes theorem [8], is not well suited since its computational performance is too degraded with fairly non-structured panel meshes. In order to overcome this shortcoming, we propose in this work an alternative way using a weak form of the Stokes theorem. This weak form allows us to compute smoothed surface gradient fields, for both doublet and source layers (or dipolar and monopolar ones, respectively) which are employed in Morino's formulation and is oriented to fairly non-structured panel meshes.

\section{Potential formulation review}

Consider an exterior flow problem, see Fig. 1, where $\Omega$ is the exterior region to the wetted surface $\Gamma_{\mathrm{S}}$ of a stream-like body. The $x$-axis is parallel to the non-perturbed velocity $\mathbf{u}_{\infty}$, the $z$-axis positive upwards and the $y$-axis results in a right-turn sequence $(x, y, z)$. The potential velocity field is given by $\mathbf{u}=\nabla \Phi$, where $\Phi$ is the total velocity potential, which satisfies the Laplace equation in the flow region $\Omega$ and it is split as $\Phi=\mathbf{u}_{\infty}^{\mathrm{T}} \mathbf{x}+\phi$, where $\mathbf{x}=(x, y, z)$ is the position vector and $\phi$ is the perturbation velocity potential. The kinematic boundary conditions are the slip condition $\partial_{n} \Phi=0$ at the wetted body surface while, at the infinity, the perturbation velocity potential tends to zero for external 


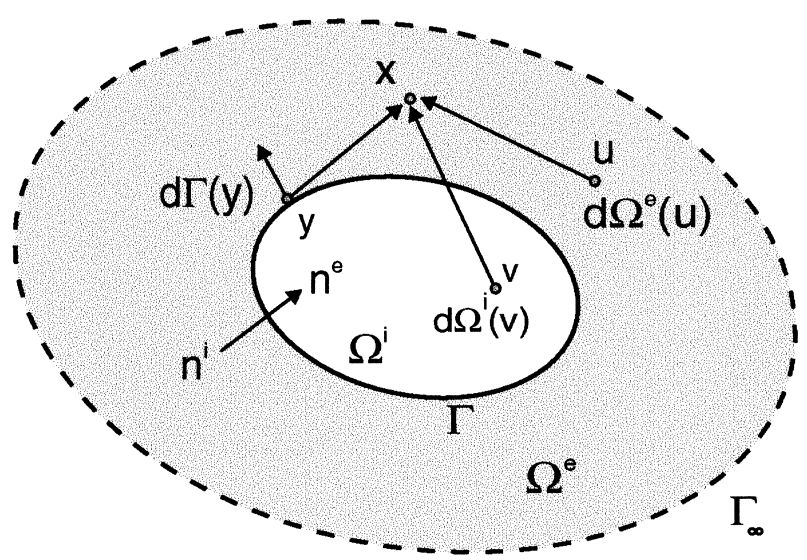

Fig. 1. Geometry for exterior flow problems.

flows, i.e. $\phi(\mathbf{x}) \rightarrow 0$ for $|\mathbf{x}| \rightarrow \infty$. Then, the governing equations for the solution $\Phi$ of this potential flow model are:
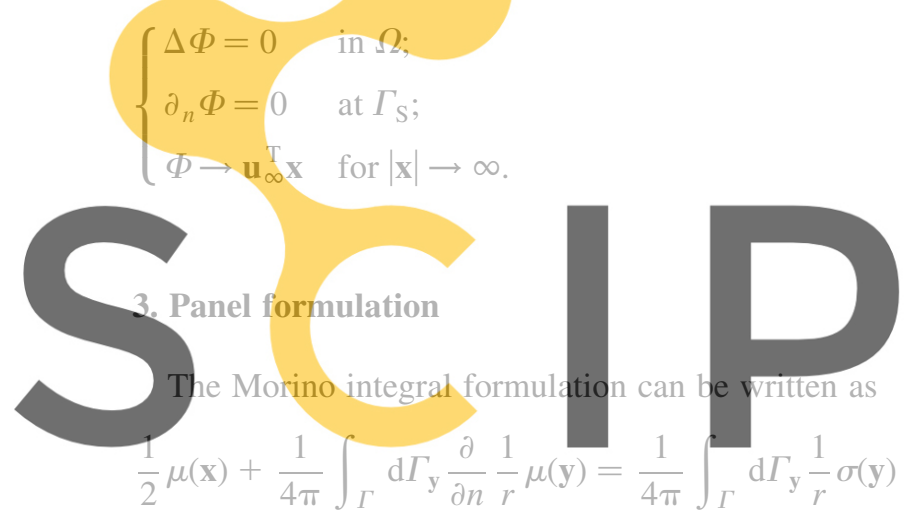

Register for free at https//www.scipedia.com to

with

$\sigma=\left(-\mathbf{u}_{\infty}, \mathbf{n}\right)$

where $\mu$ and $\sigma$ are the double and single layer densities over the surface $\Gamma, r=|\mathbf{x}-\mathbf{y}|$ is the Euclidean distance between the field point $\mathbf{x}$ and the source point $\mathbf{y}$, and $\mathbf{n}$ is the (exterior) unit normal. We discretize it using a low-order panel method. The mesh employs a low-order representation of the body surface by means of a polyhedral with $n$ flat surfaces over the wetted body surface. We impose the discretized boundary integral equation by collocation at the

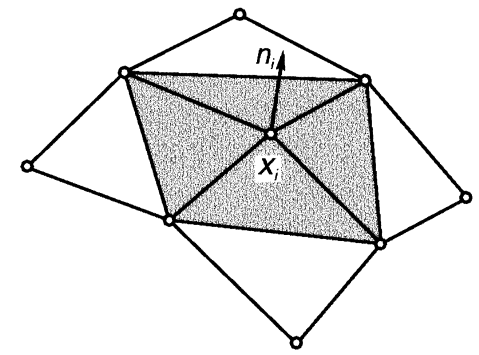

centroids of the panels, to set up the discrete lineal system $\mathbf{A} \boldsymbol{\mu}=\mathbf{b}$, where $\mathbf{A}$ is the (bipolar) matrix system and $\boldsymbol{\mu}$ is the bipolar vector evaluated at the centroids of the $n$-panels, i.e. $\boldsymbol{\mu}=\left[\mu\left(\mathbf{x}_{1}\right) \ldots \mu\left(\mathbf{x}_{n}\right)\right]^{\mathrm{T}}$, and $\mathbf{b}$ is the source vector. The source vector $\mathbf{b}=\mathbf{C \sigma}$, is the product of the monopolar influence matrix $\mathbf{C}$ and the flow vector $\boldsymbol{\sigma}=$ $\left[\sigma\left(\mathbf{x}_{1}\right) \ldots \sigma\left(\mathbf{x}_{n}\right)\right]^{\mathrm{T}}$, obtained by means of the slip boundary condition on the solid walls, that is, $\sigma_{j}=-\mathbf{u}_{\infty, j}^{\mathrm{T}} \mathbf{n}_{j}$, where $\mathbf{n}_{j}$ is the panel normally oriented to the wetted side. The bipolar and monopolar influence matrices $A_{i j}, C_{i j}$ are given by the surface integrals

$A_{i j}=\frac{1}{4 \pi} \int_{\Gamma} \mathrm{d} \Gamma_{j} \frac{\mathbf{r}_{i j}^{\mathrm{T}} \mathbf{n}_{j}}{r_{i j}^{3}}$ and $C_{i j}=\frac{1}{4 \pi} \int_{\Gamma} \mathrm{d} \Gamma_{j} \frac{1}{\left|\mathbf{r}_{i j}\right|}$

for $i, j=1,2, \ldots, n$;

where $\mathbf{r}_{i j}=\left|\mathbf{x}_{i}-\mathbf{x}_{j}\right|$ is the Euclidean distance between the centroid $\mathbf{x}_{i}$ and the integration point $\mathbf{x}_{j}$ over the $j$-panel surface with $\mathbf{x}=(x, y, z)$. These integrals can be evaluated in a closed form following an analogous procedure proposed by Medina and Liggett [9], e.g. see also D'Elía [13,14]. Both influence matrices $\mathbf{A}$ and $\mathbf{C}$ are dense and nonsymmetric and its vectorial computation can be obtained


and, then, it is evaluated the as discrete operators $\mathbf{A}=$ $\left[\mathrm{a}_{1} \ldots \mathrm{a}_{n}\right]$ and $\mathrm{C}=\left[\mathbf{c}_{1} \ldots \mathrm{c}_{n}\right]$.

\section{download the version without the watermark}

\section{Surface velocity computation}

The velocity field $\mathbf{u}$ on the body surface $\Gamma$, is obtained by means of

$\mathbf{u}=\mathbf{u}_{\infty}+\mathbf{u}^{\prime}+\mathbf{u}^{\prime \prime} \quad$ at $\Gamma$

where $\mathbf{u}^{\prime}$ and $\mathbf{u}^{\prime \prime}$ are the monopolar and dipolar velocities, respectively. They are found from (e.g. see Ref. [7])

$\left\{\begin{array}{l}\mathbf{u}^{\prime}=\sigma \mathbf{n} \\ \mathbf{u}^{\prime \prime}=-\nabla \mu\end{array}\right.$

Fig. 2. $A_{i}$-panel patch around the $i$-node. 


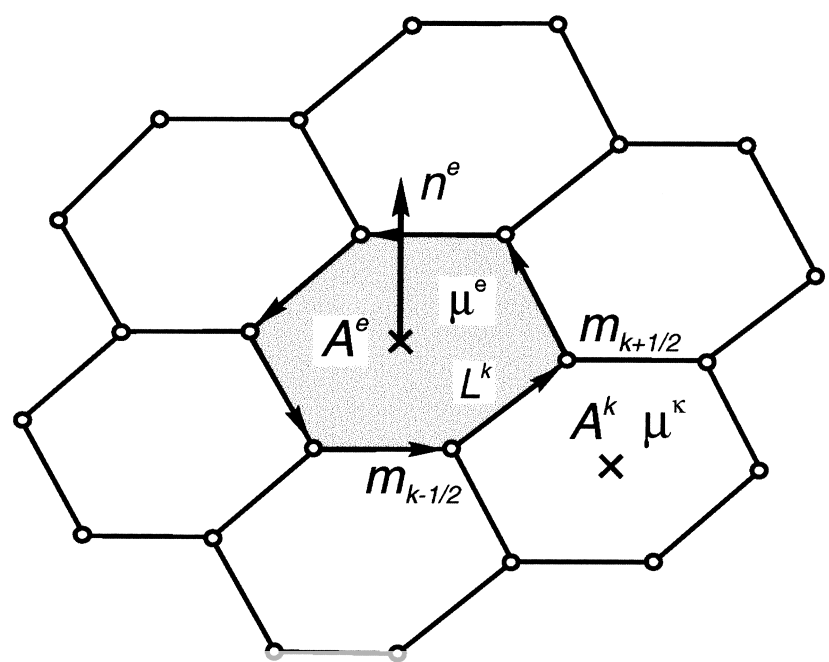

Fig. 3. Panel dipolar gradient by means of the Stokes theorem.

where $\nabla$ is the surface gradient and $\mathbf{n}$ is the unit normal to the surface. The dipolar layer $\mu$ is found solving the boundary integral Eq. (2), and the monopolar layer $\sigma$ is from the slip boundary Eq. (3). In a low order panel method, both of them are piecewise-constant on the panel mesh. A nodal velocity computation can be obtained averaging vver the $A_{i}$-panel patch adjacent to the $i$-node, as is shown in $\mathrm{F}$ 2. We show a strong (classical) form and a weak one for the perturbation velocities $\mathbf{u}^{\prime}$ and $\mathbf{u}^{\prime \prime}$, where the weak form considered in this work is developed only for triangular
panels.

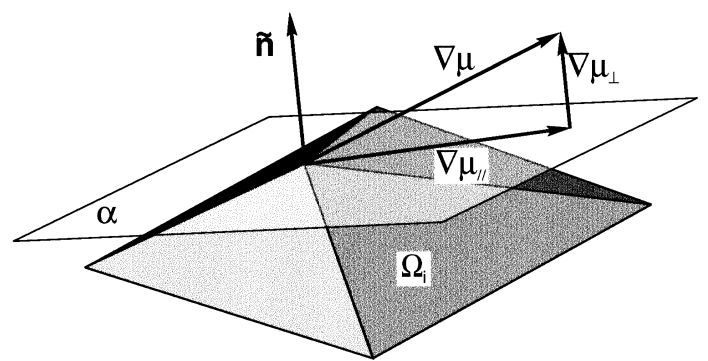

Fig. 5. Spurious component $\nabla \mu_{\perp}$ in the weak dipolar gradient.

with

$A=\sum_{e=1}^{n_{\mathrm{p}}} A^{e}$

where $A^{e}$ is the panel area, $n_{\mathrm{p}}$ is the number of panels on the $A_{i}$-panel patch and

$\mathbf{u}_{\sigma}^{e}=\sigma^{e} \mathbf{n}^{e}$

is the monopolar velocity on the $e$-panel, being $\sigma^{e}=$ $\left(-\mathbf{u}_{\infty}, \mathbf{n}^{e}\right)$ and $\mathbf{n}^{e}$ the surface density and the (constant)

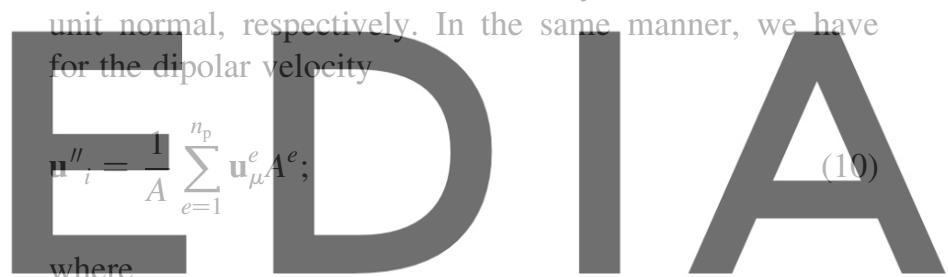



A simple average of both velocities $\mathbf{u}_{i}^{\prime}$ and $\mathbf{u}_{i}{ }_{i}$ can be obtained by using a surface average over the $A_{i}$-panel patch around the $i$-node. For the monopolar one, we write

$\mathbf{u}_{i}^{\prime}=\frac{1}{A} \sum_{e=1}^{n_{\mathrm{p}}} \mathbf{u}_{\sigma}^{e} A^{e}$

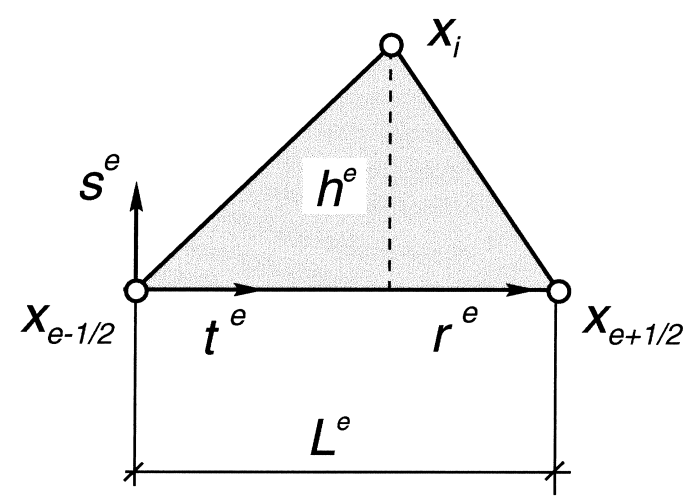

Fig. 4. Geometrical description for the shape function $N_{i}^{e}$ over a triangle panel. is the average dipolar velocity over the $e$-panel (constant) and $\nabla$ is the surface gradient. For its computation, we employ the vectorial form of the Stokes theorem (e.g. see Refs. [7,8])

$\int_{A^{e}}\left(\mathbf{n}^{e} \times \nabla \mu^{e}\right) \mathrm{d} A=\int_{L^{e}} \mu^{e} \mathrm{~d} \mathbf{L} ;$

where $L^{e}$ is the contour of the panel surface $A^{e}$ and $\mathbf{n}^{e}$ is its unit normal. When both the dipolar gradient $\nabla \mu^{e}$ and the unit normal $\mathbf{n}^{e}$ are constant over the panel surface $A^{e}$, this

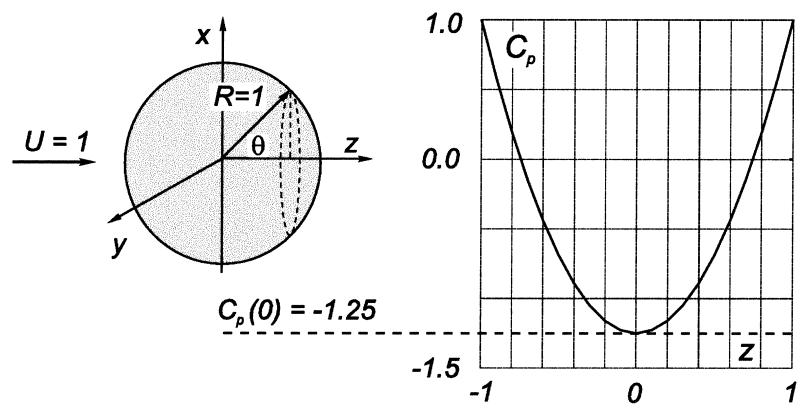

Fig. 6. Potential flow around a sphere (left), and analytical pressure coefficient (right). 


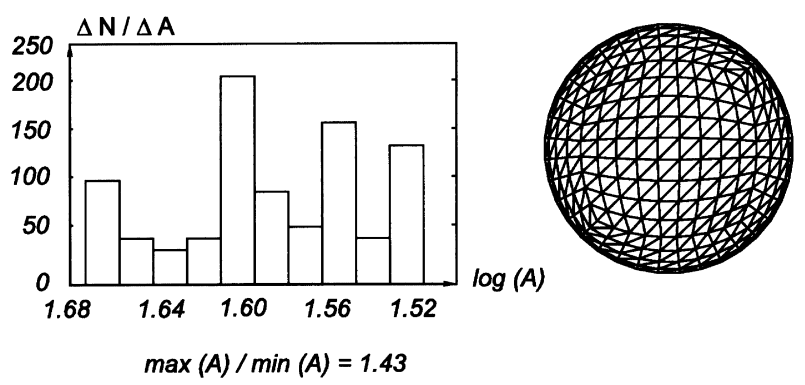

Fig. 7. Sphere with a structured mesh (right), and panel area histogram (left).

reduces to evaluate

$\nabla \mu^{e}=-\frac{\mathbf{n}^{e}}{A^{e}} \times \int_{L^{e}} \mu^{e} \mathrm{~d} \mathbf{L}$

which can be discretized, over an $e$-panel of $n_{\mathrm{s}}$ sides, see

Fig. 3, as

$\nabla \mu^{e}=-\frac{\mathbf{n}^{e}}{A^{e}} \times \sum_{k=1}^{n_{\mathrm{s}}} \tilde{\mu}^{k} \mathbf{L}^{k}$

where $\mathbf{L}^{k}$ is its $k$-side and $\tilde{\mu}^{k}$ is some average value, for
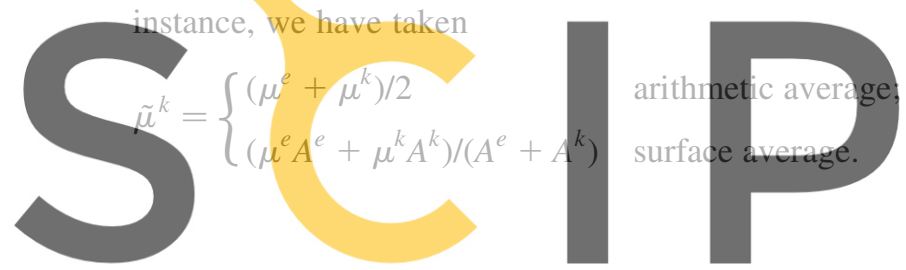

(15) way that

$N_{i}^{e}(\mathbf{x})= \begin{cases}1 & \text { for } \mathbf{x}=\mathbf{x}_{i} ; \\ 0 & \text { for } \mathbf{x}=\mathbf{x}_{e-1 / 2}+\mathbf{t}^{e} \lambda\end{cases}$

where $\lambda$ is a free parameter and

$\mathbf{t}^{e}=\frac{\mathbf{r}^{e}}{\left|\mathbf{r}^{e}\right|}$

is the unit tangent vector along the $e$-side opposite to the $i$ node and

$\mathbf{r}^{e}=\mathbf{x}_{e+1 / 2}-\mathbf{x}_{e-1 / 2}$

is the edge vector, where $\mathbf{x}_{e+1 / 2}$ and $\mathbf{x}_{e-1 / 2}$ are its vertices of the $e$-side opposite to the $i$-node. That is, the chosen shape elemental function $N_{i}^{e}$ has a unit value on the $i$-node, null over the opposite side and a linear dependence in the interval between them and, then, its gradient over the $e$-panel is constant and equals to

$\nabla N_{i}^{e}=\frac{1}{h^{e}} \mathbf{s}^{e}$

where $h^{e}$ is the panel height, $s^{e}$ is the unit vector obtained as



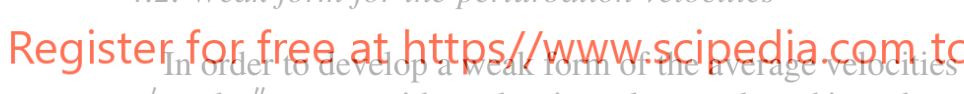

$\mathrm{u}_{i}^{\prime}$ and $\mathbf{u}_{i}^{\prime \prime}$, we consider only triangular panels and introduce the nodal shape function for the $i$-node (in the FEM sense)

$N_{i}=\sum_{e=1}^{n_{\mathrm{p}}} N_{i}^{e}$

where $N_{i}^{e}$ is the elemental shape function of the $e$-triangular panel on the $A_{i}$ panel patch. This function is chosen in such a

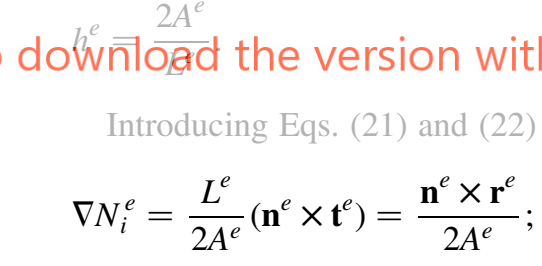

where also $\mathbf{r}^{e}=L^{e} \mathbf{t}^{e}$. A weak dipolar gradient can be obtained with the nodal shape function $N_{i}$ and the standard Galerkin procedures [10]. Let us consider the weighted

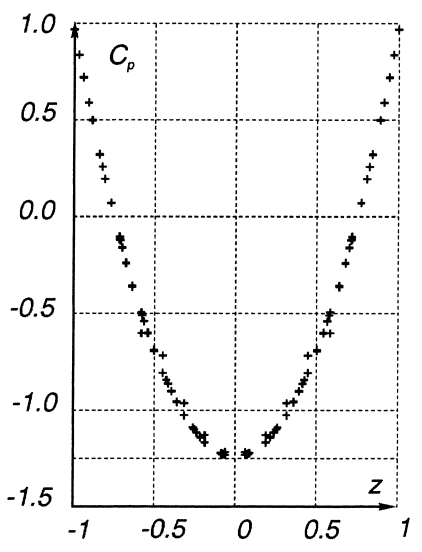

Fig. 8. Pressure coefficient $C_{\mathrm{p}}(z)$ for a structured panel mesh over a sphere using a strong form (left) and a weak one (right), respectively. 


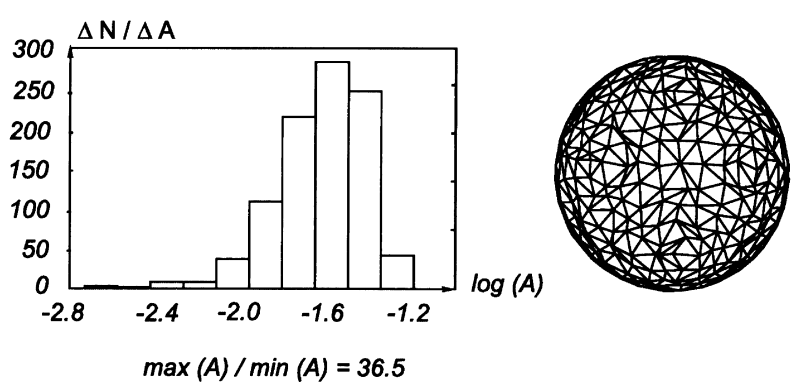

Fig. 9. Panel perturbed mesh over a sphere (right), and panel area histogram (left).

average over the $A_{i}$-panel patch

$\tilde{\nabla} \mu_{i}=\frac{\gamma_{i}}{M_{i}}$

where

$M_{i}=\sum_{e=1}^{n_{\mathrm{s}}} M_{i}^{c}$

$M_{i}^{e}=\int_{A_{i}} N_{i} \nabla \mu \mathrm{d} A$

(26)


where $N_{i}$ is linear and $A^{e}$ is a triangular element. Therefore,

$\sum_{e=1}^{n_{\mathrm{p}}} \int_{A^{e}} N_{i}^{e} \mathrm{~d} A=\frac{1}{3} \sum_{e=1}^{n_{\mathrm{p}}} A^{e}$

that is

$M_{i}=\frac{A_{i}}{3}$

introducing Eqs. (23) and (32) into Eq. (24), we arrive to the weak dipolar gradient

$\tilde{\nabla} \mu_{i}=\frac{-3}{2 A_{i}} \sum_{e=1}^{n_{\mathrm{s}}} \mu^{e}\left(\mathbf{n}^{e} \times \mathbf{r}^{e}\right)$.

\subsection{Spurious weak component}

In general, the weak dipolar gradient $\tilde{\nabla} \mu_{i}$ obtained with Eq. (33), besides a tangential component $\tilde{\nabla}_{\|} \mu_{i}$ on the discrete tangent plane at the node, contains a spurious component $\tilde{\nabla}_{\perp} \mu_{i}$ perpendicular to the tangent plane, see Fig. 5, that is,

$\tilde{\nabla} \mu_{i}=\tilde{\nabla}_{\|} \mu_{i}+\tilde{\nabla} \mu_{i}$

(34)

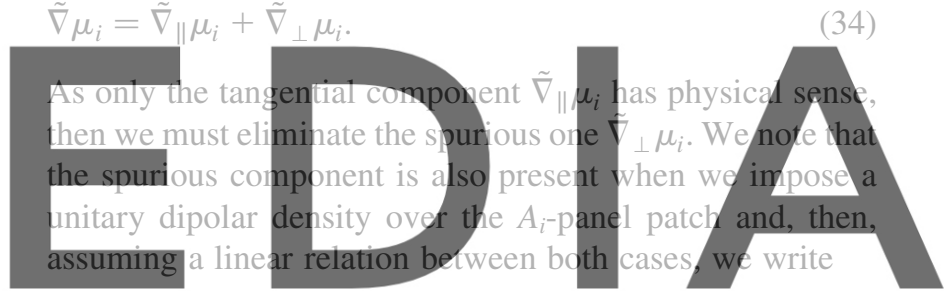

$\int \tilde{\nabla}_{\perp} \mu_{i}=\alpha_{i} \tilde{\nabla} \mu_{i}^{1}$

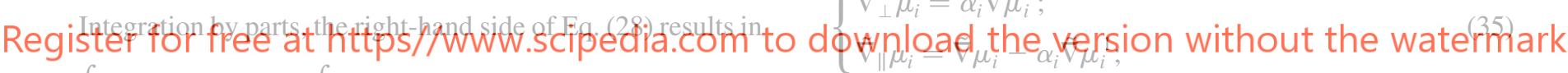

$\int_{A^{e}} N_{i}^{e} \nabla \mu^{e} \mathrm{~d} A=-\int_{A^{e}} \mu^{e} \nabla N_{i}^{e} \mathrm{~d} A ;$

It is well known in the finite element context [11], the following identity

$\int_{A^{e}} N_{i}^{e} \mathrm{~d} A=\frac{A^{e}}{3}$

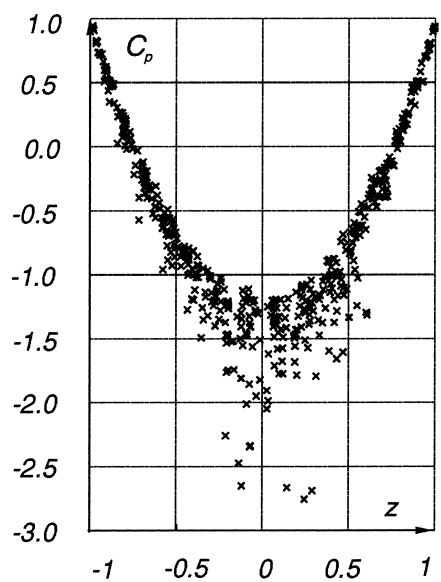

where $\alpha_{i}$ is a nodal proportionality factor, and $\tilde{\nabla} \mu_{i}^{1}$ is the unitary dipolar gradient obtained by means of Eq. (33), when $\mu_{i}=1$ is imposed on the $A_{i}$-panel patch, resulting in

$\tilde{\nabla} \mu_{i}^{1}=\frac{-3}{2 A_{i}} \sum_{e=1}^{n_{\mathrm{s}}}\left(\mathbf{n}^{e} \times \mathbf{r}^{e}\right)$;

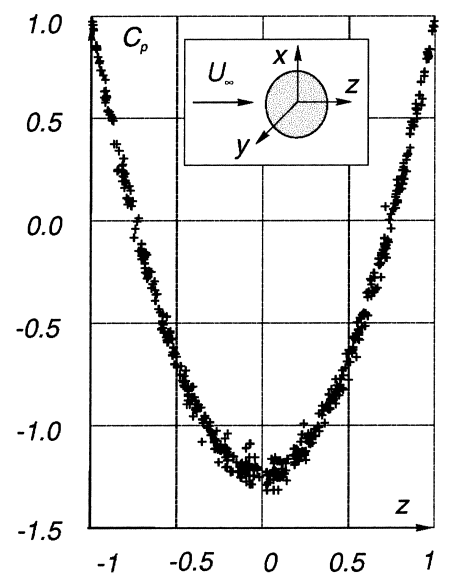

Fig. 10. Pressure coefficient $C_{\mathrm{p}}(z)$ for a non-structured panel mesh over a sphere using a strong form (left) and a weak one (right), respectively. 

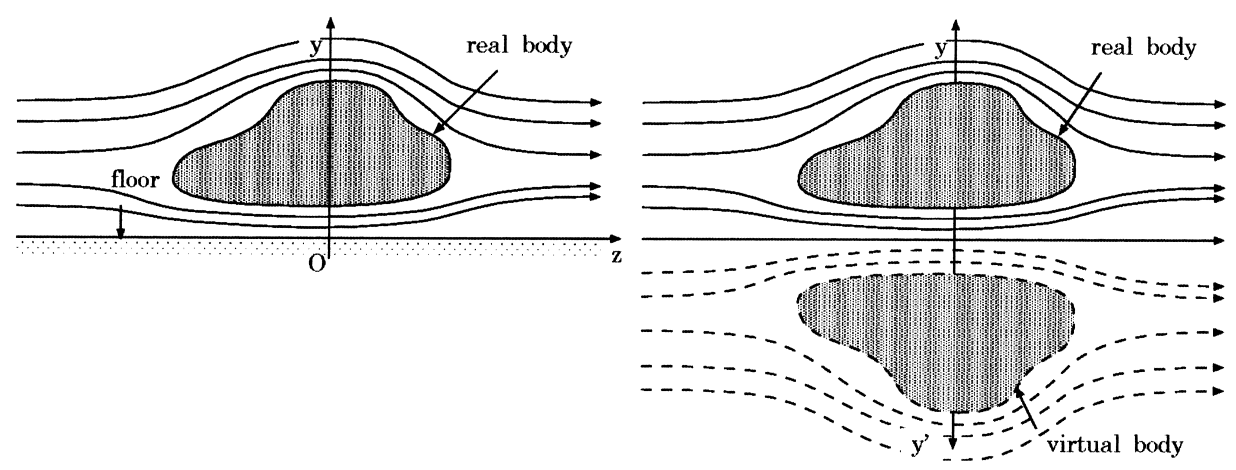

Fig. 11. Sketch for the attached potential flow around a car-like body with the floor's influence.

which is orthogonal to the tangential one

$\left(\tilde{\nabla} \mu_{i}^{1}, \tilde{\nabla}_{\|} \mu_{i}\right)=0$.

Replacing Eq. (35) into Eq. (37) we find
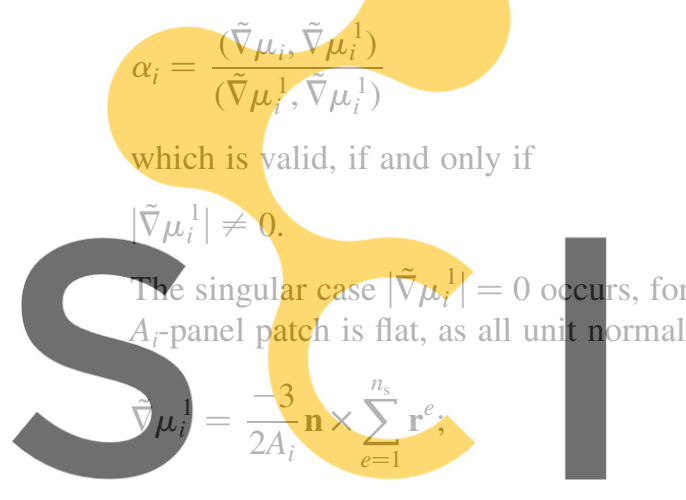

whereas

Register for free at https//www.scipedia.com to $\sum_{e=1} r^{e}=0$

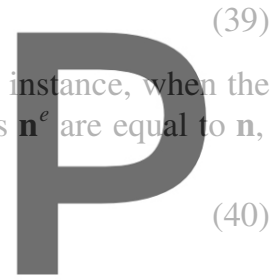

since it is a vectorial closed sum. Nevertheless, in this case, Eq. (33) can be used for plane patches. A further useful consequence of the procedure proposed to eliminate the spurious component, is the weak normal unit vector

$\tilde{\mathbf{n}}_{i}=\frac{\tilde{\nabla} \mu_{i}^{1}}{\left|\tilde{\nabla} \mu_{i}^{1}\right|}$.

In brief, the weak monopolar and dipolar velocities are found from

$\left\{\begin{array}{l}\tilde{\mathbf{u}}_{i}{ }_{i}=\sigma_{i} \tilde{\mathbf{n}}_{i} ; \\ \tilde{\mathbf{u}}^{\prime \prime}{ }_{i}=-\tilde{\nabla}_{\|} \mu_{i} ;\end{array}\right.$

if the condition (39) is fully verified.

\section{Numerical examples}

\subsection{A sphere}

In order to show the suitability of the proposed method, we have considered the flow around a sphere of radius $R=1$ with non-perturbed speed $U=1$. The analytical solution for the surface velocity $u(\theta)$ is $[12]$

$u(\theta)=(3 / 2) U \sin (\theta)$

and the pressure coefficient $C_{\mathrm{p}}(\theta)$ is found from

$C_{\mathrm{p}}(\theta)=1-\frac{u(\theta)^{2}}{U^{2}} ;$

we have, see Fig. 6,

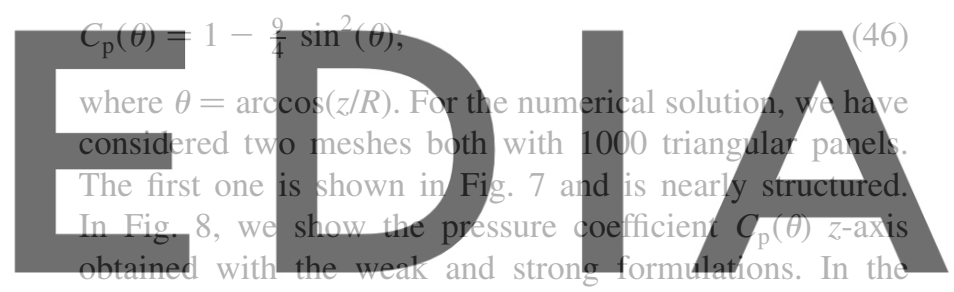

last case, we can see a small tendency toward dispersion,

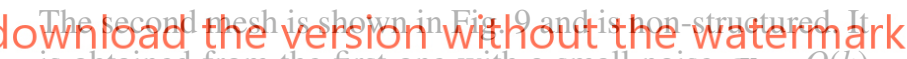
is obtained from the first one with a small noise $\mathbf{e X}=O(h)$


Fig. 12. Car-like meshes with 3100 panels: a structured mesh (left) and a non-structured one (right). 

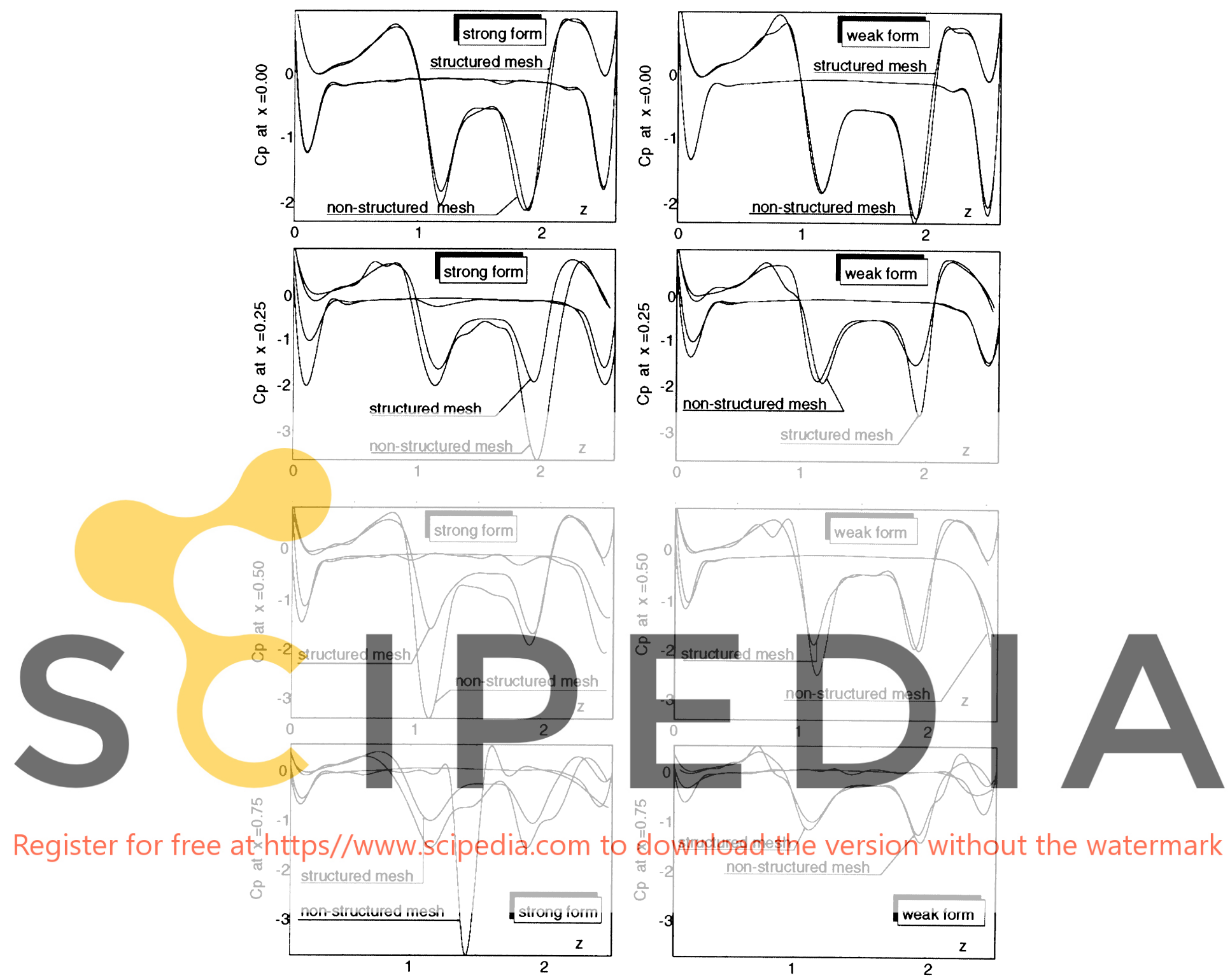

Fig. 13. Pressure coefficient $C_{\mathrm{p}}(z)$ over a car-like body, on section cuts at $x / B=0,1 / 4,1 / 2,3 / 4$, for structured and non-structured meshes of 3100 panels, as a strong form (left) and a weak one (right).

added to the nodal coordinates, where $h$ is an average diameter of the panels, then we have re-projected to the unit sphere, verifying that the panels do not degenerate. In Fig. 10, we show the pressure coefficient $C_{\mathrm{p}}(\theta)$ along the $z$-axis obtained with both formulations, where the dispersion tendency of the strong form is evident while the weak form is clearly more robust.

\subsection{Car-like configuration}

We have considered the non-separated potential flow around a 3D car-like body with the floor influence, as sketched in Fig. 11. As a first approach, the floor influence could be taken into account with a panel mesh over a large but finite floor panel mesh, but this strategy introduces an error due to the plane boundary effects and also the computational cost will be expensive as larger planes are considered. In order to overcome this short-coming, we observe that the flow regime is equivalent to consider a mirrored configuration with respect to the floor (real body + image). In such a case, we have a symmetric virtual car-like body and it is not necessary to introduce the plane. We, then, have a total number of panels, which is twice the number of panels on the body. But, due to the symmetry of the flow, the unknown potentials on the image can be eliminated in terms of the potentials on the body, and the resulting CPU time is almost the same as for the isolated body. We performed numerical experiments with a (smooth) structured mesh and a non-smooth topologically structured one (in brief: structured and non-structured meshes, 
respectively). The panel mesh, for each mesh type, has 3100 triangular panels, see Fig. 12. The symmetry of the velocity potential with respect to the floor also allows us to reduce the effective dimension of the linear system. The pressure coefficients $C_{\mathrm{p}}(z)$ are shown in Fig. 13, on section cuts, at $x / B=0,1 / 4,1 / 2,3 / 4$, the strong form (left-hand side) is much more sensitive than the weak one (right-hand side).

\section{Conclusions}

We have considered a weak form for the computation of the dipolar and monopolar surface gradients related to a low-order panel method. The flow problem was formulated by means of a 3D potential model and the method of discretization was based on Morino's formulation for the perturbation velocity potential. On the body surface this representation was reduced to an integral equation with source and the doublet densities. The first one was found by application of the boundary condition, and the second one is the unknown over the surface of the body. A loworder panel method was used for the analytic integrations of the monopolar and dipolar influence coefficients. Then, a weak form of the Stokes theorem was developed for the

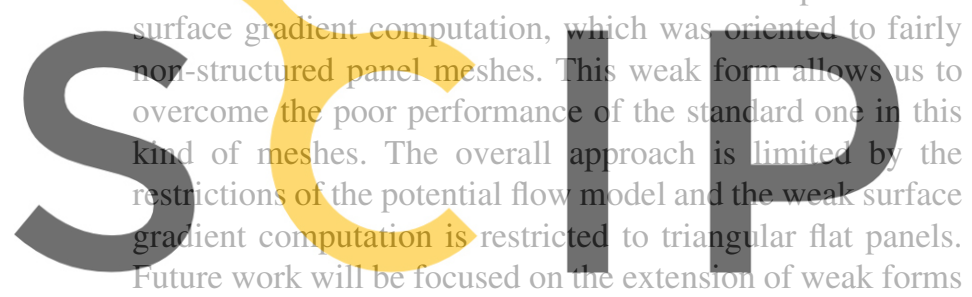

to panels with an arbitrary number of sides or to high-order Registenefdoprfneedicatb. https//www.scipedia.com to

\section{Acknowledgements}

This work has received financial support from Consejo
Nacional de Investigaciones Científicas y Técnicas (CONICET, Argentina) and Banco Interamericano de Desarrollo (BID) through grant BID 802/OC-AR PID Nr. 26, and from Universidad Nacional del Litoral (Argentina). We made extensive use of Free Software Foundation/GNU-Project resources.

\section{References}

[1] Morino L, Kuo CC. Subsonic potential aerodynamics for complex configurations: a general theory. AIAA Journal 1974;12:191-7.

[2] Katz J, Plotkin A. Low-speed aerodynamics, from wing theory to panel methods. New York: McGraw-Hill, 1991.

[3] Mokry M. Complex variable boundary element method for external potential flows, 28th Aerospace Sciences Meeting, January 8-11, Reno, NV, 1990

[4] Storti M, D'Elía J, Idelsohn S. CVBEM formulation for multiple profiles and cascades. App Mech Rev Part 2 1995;48(11):203-10.

[5] Hess JL. Review of the historical development of surface source methods. In: Morino L, editor. Computational methods in potential aerodynamics, Berlin: Springer, 1985

[6] Morino L, editor. Computational methods in potential aerodynamics Berlin: Springer, 1985

[7] Maître T. Modelisation de l'ecoulémént autour d'une hélice marine par la méthode du potentiel. Tesis Doctoral, Institut National Poly-

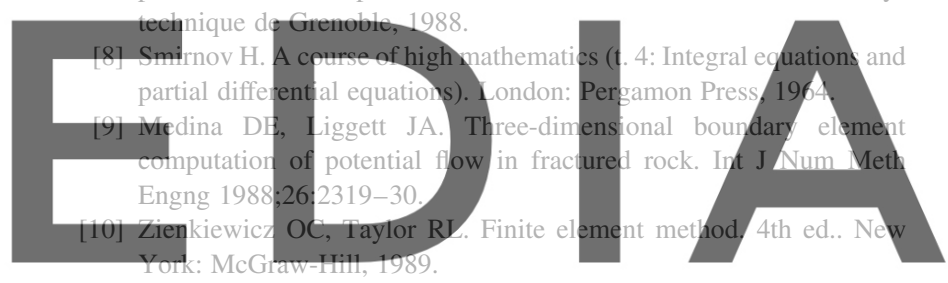

[11] Segerlind L. Applied finite element analysis. New York: Wiley, 1983. downlRad the yersion without the watermark

[13] D’Elía J, Storti M, Idelsohn S. A closed form for low-order panel methods. Advances in Engineering Software 2000;31:347-53.

[14] D'Elía J, Storti M, Idelsohn S. Iterative solution of panel discretizations for potential flows. The modal/multipolar preconditioning. Int $\mathrm{J}$ Num Meth Fluids 2000;32:1-2. 